\title{
Pedunculated Hepatocellular Carcinoma: Distinguishing Features from Conventional Hepatocellular Carcinoma
}

\author{
Norzila $A B^{\mathrm{a}}$, Wong $M \mathrm{MK}^{\mathrm{b}}$ \\ ${ }^{a}$ Department of Radiology, School of Medical Sciences, Universiti Sains Malaysia (USM), 16150 Kubang \\ Kerian, Kelantan, Malaysia. \\ ${ }^{b}$ Advanced Medical and Dental Institute, Universiti Sains Malaysia, Persiaran Seksyen 4/9, \\ Bandar Putra Bertam, 13200 Kepala Batas, Pulau Pinang, Malaysia
}

\begin{abstract}
Malignant pedunculated (exophytic) hepatocellular carcinoma (P-HCC) is a rare entity of hepatocellular carcinoma. Due to its unique characteristics, it gives a different spectrum of clinical findings, radiological presentation and different management approach with reported better outcome to the patients. We present a case report of a lady diagnosed with malignant pedunculated hepatocellular carcinoma.
\end{abstract}

Keywords: Pedunculated hepatocellular carcinoma; Exophytic hepatocellular carcinoma

\section{INTRODUCTION}

Malignant pedunculated (exophytic) hepatocellular carcinoma (P-HCC) has been reported to occur in $0.24 \%-3.0 \%$ of all HCC patients. ${ }^{1}$ P-HCC is a rare entity comprising about $0.2-4.2 \%$ of all hepatocellular carcinoma. It gives a different spectrum of clinical findings, radiological presentation and different management approach with better outcome to the patients. Our patient was diagnosed with pedunculated hepatocellular carcinoma that slightly deviates from its usual presentation, which serves many interesting points for learning and discussion.

\section{CASE REPORT}

Our patient is a 43-year old Malay lady with background history of Hepatitis B presented with intermittent left hypochondriac and epigastric pain. Clinically per abdomen, a $15.0 \mathrm{~cm} \times 15.0 \mathrm{~cm}$ mass was palpable at the left hypochondriac area with ascites and left pleural effusion. Ultrasound

Corresponding author:

Dr Norzila Tendot Abu Bakar

Department of Radiology, School of Medical

Sciences,

Universiti Sains Malaysia (USM), 16150 Kubang

Kerian,

Kelantan, Malaysia.

Office: 09-7673468, HP: 012-2886284 abdomen (Figure 1) showed a large heterogenous highly vascular epigastric mass in-continuity with inferior margin of left liver lobe with multiple area of necrosis within. Multiple small hypoechoeic lesions were scattered in the liver parenchyma which has a nodular margin. A diagnosis of hepatocellular carcinoma with liver metastasis was anticipated.

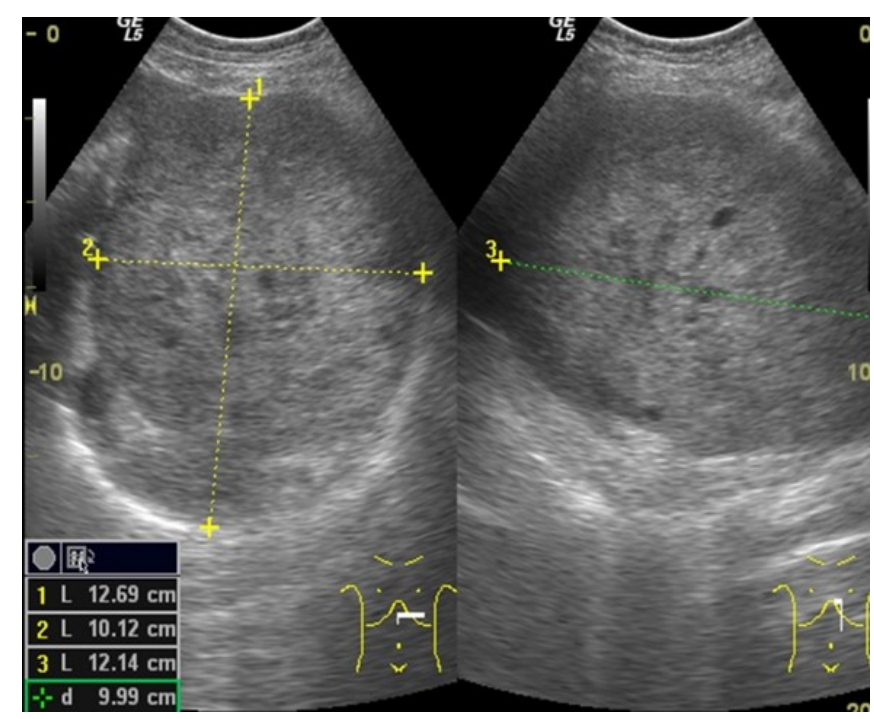

Figure 1. Ultrasound picture showing a heterogenous hyperechoiec mass in the epigastrium.

Her blood investigations showed an extremely high alpha-fetoprotein level of 118, $292 \mathrm{ng} / \mathrm{mL}$ with slightly low level of serum albumin of $28.0 \mathrm{~g} / \mathrm{dL}$. Liver enzymes were within normal the limit. 
Contrasted CT Abdomen (Figure 2 and 3) showed a large well-defined pedunculated heterogeneously enhancing mass with central necrosis arising from the inferior part of left liver lobe, measuring $12.4 \mathrm{~cm}$ (AP) $\times 11.4 \mathrm{~cm}(\mathrm{~W}) \times 15.0 \mathrm{~cm}(\mathrm{CC})$. This mass was very vascular with the main feeder is from right hepatic artery. It pushes the stomach medially and the spleen inferiorly. Small hypodense lesion was seen in segment VIII of the right liver lobe. Diagnosis of pedunculated (exophytic) hepatocellular carcinoma was made.

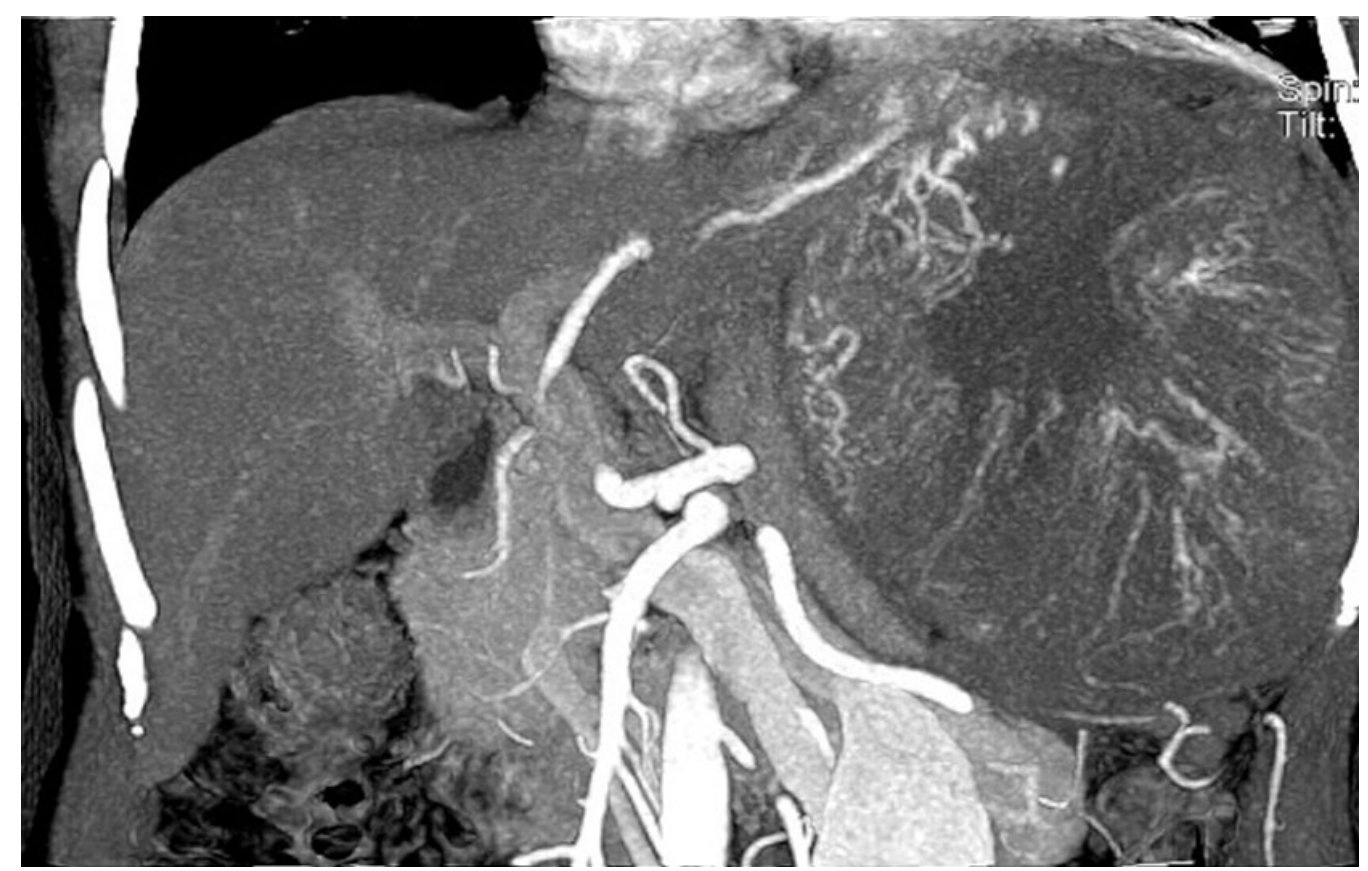

Figure 2. CECT Abdomen (in arterial phase) showing large pedunculated

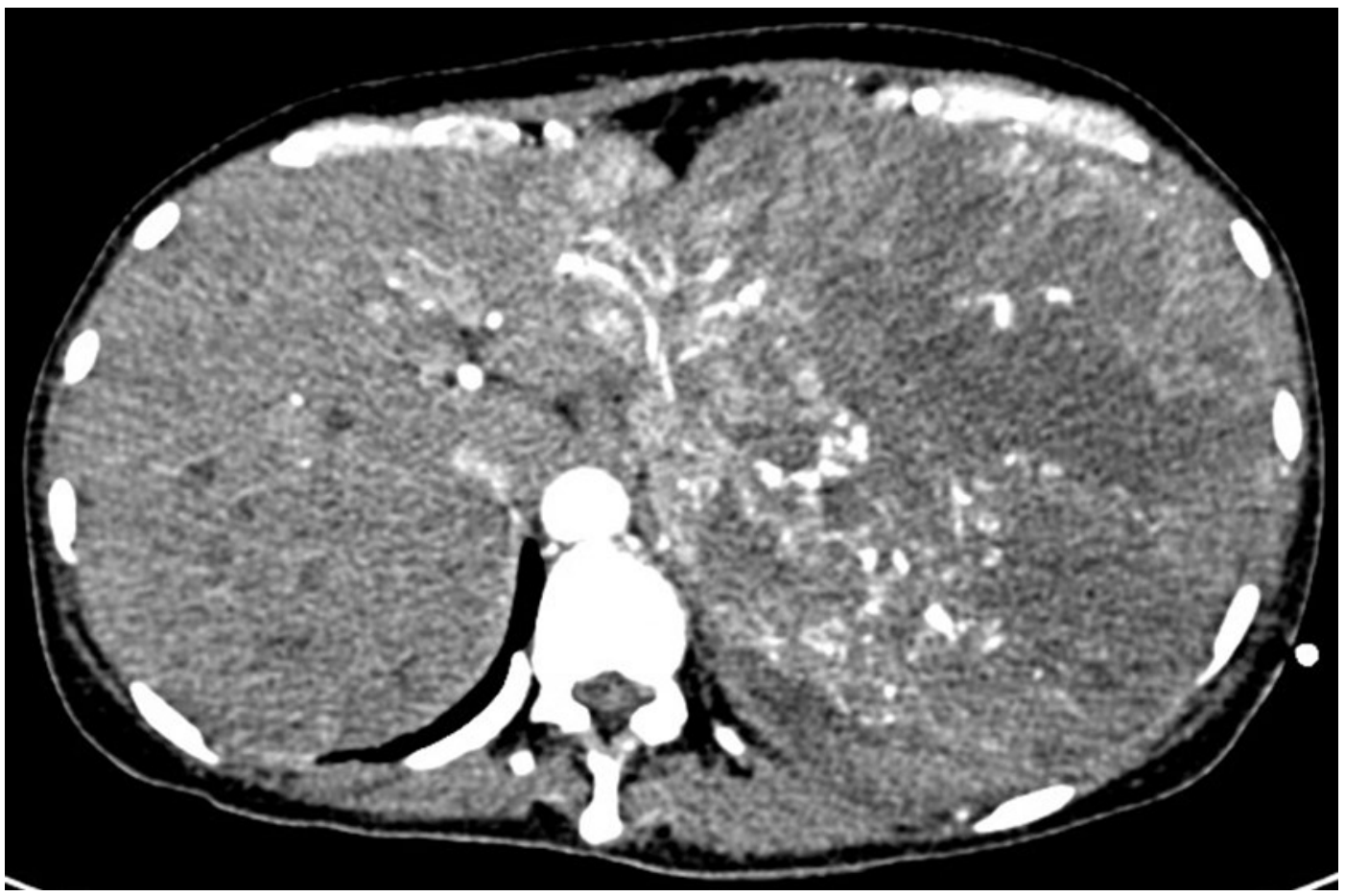

Figure 3. Multiple liver nodules seen in segment VII and VIII. 
Correlating with extremely high alpha fetoprotein, a diagnosis of exophytic hepatocellular carcinoma with lung and liver metastasis were entertained. Ultrasound guided biopsy confirmed the CT scan findings of hepatocellular carcinoma.

Exploratory laparotomy and debulking surgery was performed. A large mass enwrapped by the peritoneum which was densely adhered to the left liver lobe was removed together with the omental nodules measuring $3 \mathrm{~cm} \times 3 \mathrm{~cm}$ in size. Histopathological investigations of the left hypochondriac mass and omental nodule confirmed the findings of hepatocellular carcinoma (Trabecular type).

\section{DISCUSSION}

Hepatocellular carcinoma (HCC) occasionally grows outside the liver into the abdominal cavity. It is specifically termed extrahepatic growth of HCC or pedunculated $\mathrm{HCC}$ or exophytic HCC. ${ }^{1,2}$ Pedunculated hepatocellular carcinoma (P-HCC) which protrudes the liver with or without pedicle was first described by Roux in 1897.,4 Until 2002, only 173 cases were reported in the literature. Its incidence was reported to be $0.24 \%-3.0 \%$. It was reported that $\mathrm{P}-\mathrm{HCC}$ are more common in men $(73 \%)$ as compared to women $(27 \%)$, and affecting people at age of 33-80 years old. ${ }^{5}$ The predisposing factor of developing P-HCC is similar to other type of hepatocellular carcinoma.

P-HCC can arise from various part of liver. The most common site is right liver lobe, followed by left liver lobe and from inferior hepatic rim. Several theories have been discussed in regard to the origin of $\mathrm{P}$ HCC. It was postulated that P-HCC can arise from congenitally displaced lobule in capsule of Glisson. Others postulated that P-HCC arise from accessory liver lobe, because this lobe is small and can project in any direction. ${ }^{6}$ It also can project through defect in anterior abdominal wall. Furthermore, accessory liver lobe usually is found at right lobe of liver and can be attached by pedicle containing vessel and bile ducts. Although accessory lobe more common in right lobe, it can also arise from inferior hepatic margin and from any other site. Another theory is PHCC may arise from ectopic liver tissue which could be anywhere, ie triangular ligament, spleen, umbilical fossa, omentum, retroperitoneum, near celiac axis as well as gallbladder and its vicinity.

Histopathologically, our patient has trabecular type of growth, which is a well-differentiated histologic characteristics and significantly favourable overall survival compared to rest of histologic subtype. ${ }^{7}$ The pre-operative diagnosis of P-HCC was difficult., However, with recent advances in diagnostic tool such as ultrasound, CT scan and angiography, a preoperative diagnosis is now feasible. ${ }^{10}$

In P-HCC patients, the tumour size is usually more than $5 \mathrm{~cm} .{ }^{7}$ And due to its location, P-HCC's growth is usually projecting into the abdominal cavity which is relatively bigger and spacious. Therefore, P-HCC can grow without restriction resulting in its huge size. This is also a reason why $\mathrm{p}-\mathrm{HCC}$ is more amenable for curative surgery as compared to conventional HCC due to more hepatic reserve, providing that early diagnosis was made. This will also lowers the tumour recurrence rate after resection.

Compared to conventional HCC, P-HCC has more established capsule formation. Capsule formation is related to tumour biologic characteristic and usually its formation begins at early stage. The higher percentage of capsule formation will render lower incidence of tumour emboli and better tumour cell differentiation. However, capsule formation do not guarantee survival of patient.

P-HCC also noted to have less vascular invasion in related to its prominent capsule formation. Therefore, there is reduced risk of recurrence. Vascular invasion explains why intrahepatic metastasis by portal venous system is and important mechanism for intrahepatic recurrence. Owing to the less vascular invasion, there is better survival of patients with P-HCC.

Surgical mortality in patients with P-HCC is also low compared to conventional HCC. ${ }^{5}$ High operable rates is a known characteristic in patients with $\mathrm{P}$-HCC. ${ }^{10}$ It was reported that most surgically treated P-HCC patients died of distant metastasis which was not noted pre- and intra-operatively, whereas medically treated patients with P-HCC died of GIT and tumoural bleed. ${ }^{10}$ Therefore, surgical intervention resulted in prolonged survival rate in $\mathrm{P}-\mathrm{HCC}$ patients. 
Table 1. Summary of differences between conventional HCC and P-HCC

\begin{tabular}{lll}
\hline & \multicolumn{2}{c}{ Conventional HCC } \\
\hline Growth & Within liver parenchyma & Projecting outside, towards peritoneal cavity \\
Site & Anywhere & More in right lobe \\
HPE & Commonly aggressive & Well-differentiated \\
Size & $\begin{array}{l}\text { Depends on type (micronodular, large nodular or } \\
\text { differentiated) }\end{array}$ & Usually more than $5 \mathrm{~cm}$ \\
Surgery & Depends on stage of tumour & Usually amenable for curative surgery \\
Capsule & Uncommon & Common \\
Vascular & Increased & Less \\
Surgical & High & Low \\
Mortality & & \\
\hline
\end{tabular}

Patients with P-HCC usually have large tumour size more than $5.0 \mathrm{~cm}$. Tumour size played a significant role in patients' prognosis and significantly affects survival. Our patient has huge tumour mass measuring $12.4 \mathrm{~cm} \times 11.4 \mathrm{~cm} \times 15.0 \mathrm{~cm}$. Large tumour was also associated with significantly higher risk of recurrence. The influence of tumour size is attributed to many factors, including increased invasiveness and adequacy of the resection.

P-HCC shows a significantly better overall survival because it is usually encapsulated, with wide resection margin and exhibit less vascular invasion. The preoperative diagnosis of pedunculated HCC was difficult previously, but with the availability of MRI and MDCT scans, the diagnosis is not that difficult now. ${ }^{9}$

\section{CONCLUSION}

Pedunculated hepatocellular carcinoma (P-HCC) is an uncommon entity of hepatocellular carcinoma showing several different radiological spectrum from the conventional hepatocellular carcinoma.

\section{REFERENCES}

1. Horie, Y., Katoh, S., Yoshida, H., Imaoka, T., Suou, T. \& Hirayama, C. (1983) Pedunculated Hepatocellular Carcinoma. Cancer, 51: 746-

51.

2. Okuda, K., Arakawa, M., Kubo, Y., Sakata, K., Kage, M., Iwamoto, S., Takeda, S., Sonoda, K. \& Sanefuji, H. (1998) Right sided pedunculated Hepatocellular carcinoma: a form of adrenal metastasis. Hepatology. 27: $81-85$

3. Keechilat Pavithran1, Surendran Sudhindran, Hanging Liver Tumor, J Gastrointestin Liver Dis, March 2011 Vol. 20, No 1, 8 (3)
4. Mao-Lin Yan, Yao-Dong Wang, Zhi-De Lai, YiFeng Tian, Hong-Biao Chen, Fu-Nan Qiu, SongQiang Zhou Pedunculated hepatocellular carcinoma and splenic metastasis, World $\mathrm{J}$ Gastroenterol 2009 November 7; 15(41): 5239 5241 (4)

5. Yutaka Horie, Azusa Shigoku, Hisao Tanaka, Yasushi Tomie, Naoto Maeda, Ushio Hoshino, Masahiko Koda. Prognosis for pedunculated hepatocellular carcinoma. 1999. Oncology. 57: 23-28. (5)

6. Omanik S, Jablonsky I. Pedunculated accessory hepatic lobe. Arch Surg. 1972;105:792-794.

7. Yeh, CN., Lee, WC., Jeng, LB. \& Chen, MF. (2002) Pedunculated hepatocellular carcinoma: Clinicopathologic study of 18 surgically resected cases. World J. Surg. 26: 1133-1138

8. P.P. Anthony, K. James. Pedunculated hepatocellular carcinoma, is it an entity? Histopathology. 1987.11(4): 403-414

9. Cunningham PL, Nava H, Lopez C, Douglass HO., Jr Pedunculated primary hepatocellular carcinoma. J Surg Oncol. 1984;27:260-267.

10. Moritz MW, Shoji M, Sicard GA, Shioda R, DeSchryver K. Surgical therapy in two patients with pedunculated hepatocellular carcinoma. Arch Surg. 1988 Jun;123(6):772774. 